\title{
Brain Computer Interface
}

\author{
Vanshi Sharma
}

\begin{abstract}
Computer Technology is advancing day by day and with that it has led to the idea of Brain Computer interaction. Modern computers are advancing parallelly to our understanding of the human brain. This paper basically deals with the technology of BCI (Brain Computer Interface) that can capture brain signals and translate these signals into commands that will allow humans to control devices just by thinking. These devices can be robots, computers or virtual reality environment. The basis of $\mathrm{BCI}$ is a pathway connecting the brain and an external device. The aim is to assist, augment or repair human cognitive or sensory motor function. This paper also reflects light on the application areas that BCIs help in. It contributes in medical research and neuronal rehabilitation. New companies are emerging that are developing game environments involving brain computer interface.
\end{abstract}

Keywords: Actus Reus, Brain Computer Interface (BCI), Electrocortigraphy (ECoG), Electroencephalography (EEG), Mens Rea, Neuro prosthetic

\section{INTRODUCTION}

The ability to enhance our physical and mental ability no longer exists in the world of science fiction alone. BCIs are helping them come alive in our reality. Brain computer interfaces (BCI) help in controlling computers and other electronic devices with brain activity. It enables a direct communication pathway between brain and object that the user wishes to control. This technology has a considerable potential for restoration of motor behaviours for physically challenged people. People suffering from cerebral palsy, spinal cord injury, amyotrophic lateral sclerosis (ALS) and stroke will benefit greatly. Many issues prevail that preclude experimental BMIs being translated into clinical trials. Most BMIs have only been tested in experimental animals. More experiments need to be performed before BMIs are considered safe. Here, in this paper we account for the history of the field and its growth.

\section{HISTORY}

The history of BCI begins with the discovery of electrical activity of human brain by Hans Berger and development of electroencephalography (EEG). The human brain activity was recorded for the first time by Hans Berger in 1924 by an EEG. Oscillatory activities, such as alpha wave (8-13 Hz), also called as the Berger's wave were identified by Berger after analyzation of the EEG traces. His first recording device involved inserting silver wires under the patient's scalp. Later, they were replaced by silver foils. These silver foils were joined with the help of rubber bandages to the subject's head.

Revised Manuscript Received on August 15, 2020.

* Correspondence Author

Vanshi Sharma*, SCOPE, Vellore Institute of Technology, Vellore, India. E-mail: vanshi0708@gmail.com

(C) The Authors. Published by Blue Eyes Intelligence Engineering and Sciences Publication (BEIESP). This is an open access article under the CC BY-NC-ND license (http://creativecommons.org/licenses/by-nc-nd/4.0/)
These sensors were connected to the Lippmann capillary electrometer but gave disappointing results.

However, Siemens double-coil recording galvanometer that could display very small electric voltages such as one ten thousandth of a volt, it gave successful results. The term "BCI" was coined by Jacques Vidal, who is known as the inventor of BCIs among the BCI community. His first BCI involved visually evoked potentials that allowed users to control cursor direction. In 1875 Richard Canton discovered the presence of electrical signals in animal brain. It inspires Berger. In 1998, researcher Philip Kennedy implanted the first BCI into a human. In 2001, John Donoghue and his team of researchers at the Brown University formed CyberKinetics, a public traded company with the aim of designing BrainGate, a BCI. Their first product was marketed as NeuroPort. It enabled researchers identify micro-seizure activity before patients suffering from epileptic seizures. In June, 2004, Matthew Nagle was implanted with a BCI, CyberKinetics BrainGate and became the first human to do so.

\section{TYPES OF USERS}

BMIs have been focused mainly on disabled patients such as brainstem lesions, amyotrophic lateral sclerosis (ALS) etc. These people have lost their muscle control. Current BMIs require long training periods and levies high cognitive load. The user is rendered unable to perform any other activity other than interact with the BMI. BMIs find their application by augmented reality users.

\section{METHODOLOGY}

\section{A. Non-invasive}

BMIs use either invasive or non-invasive technique for electrophysical recordings. Non-invasive BMIs utilize electroencephalograms in order to control computer cursers and other gadgets. This has proved to be helpful for paralyzed patients. It has its own pros and cons. Although, it doesn't expose patients to brain surgery, its typical transfer rate is 5-25 bits/s. At such limited capacity, movements of leg or arm prosthetic is not possible but research indicates that they might be helpful in future for wheelchair control, computer operation, communication and cursor control. Experiments were conducted in the 1960s and 1970s that aimed at allowing human subjects achieve control their brain rhythms. According to Nowlis and Kamiya, human subjects could detect their alpha rhythms after training with EEG biofeedback. Later Sterman and colleagues arrived at the same conclusions with humans and cats using sensorimotor mu rhythm. BCI based on EEG decode the subject's choice by measuring the united electrical activity of huge neuron population. Resolution is lost while the signals conduct through skin, brain tissue and bone.

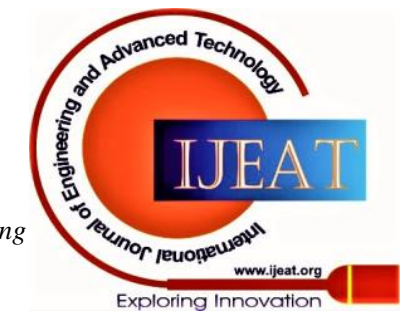




\section{Brain Computer Interface}

Despite the obstacles, EEG based methods detect brain activity corresponding with gaze angle, visual stimuli, cognitive states and voluntary intentions. One of the divisions of BCIs use Visual evoked potentials, which takes place whenever the subject views any element on the computer monitor. Slow cortical potentials that are recorded in various cortical regions and faster beta and mu rhythms that were recorded over sensorimotor cortex were utilized in BCI depictions for driving computer cursors.

Some BCIs depend on the subjects' potential to gain control of the activity of their brain utilizing biofeedback. Some make use of classifier algorithms that can identify EEG patterns connected to specific voluntary goal. Another strategy for EEG based BCIs is using virtual reality systems for providing realistic feedback for BCI training.

BCIs that utilize mu and beta rhythms were tested on paralyzed people. According to a study, a tetraplegic patient learnt to grab articles from his paralyzed hand. The BCI could detect beta waves in the patient's sensorimotor cortex, also activating an operational electrical stimulation device. P-300based BCIs when experimented on tetraplegic patients, it was observed that the patients could achieve some control over the device.

Functional magnetic resonance imaging(fMRI) has been exploited as a new technique to operate BCIs. Although they suffer temporal delays, they sample deep brain structure's activity and have good spatial resolution. Myoelectric systems that utilize voluntary activations of unaffected muscles in subjects who are partially paralyzed and amputee, utilize these signals for controlling exoskeletons and limb prostheses. They can be seen as an alternative to non-invasive BCIs.

Although no clear breakthrough has been reported in literature, they have been helpful in enhancing life of patients. One such example would be BCI for spelling. But if the aim is to achieve movement allowing multiple degrees of freedom, the task requires to record high resolution signals from brain. This can be achieved by invasive approaches.

Non-invasive BCIs have been used on patients of paralysis to achieve brain-control of upper and lower prosthetic extremity devices. Gert Pfutschellar and his colleagues at Graz University of Technology displayed BCI controlled electrical stimulation system for restoring upper extremity movements in tetraplegic patients. In 2012- 2013, researchers of University of California, Irvine showed for the first time even after a spinal cord injury BCI could restore brain- controlled walking.

\section{B. PARTIALLY INVASIVE} device is outside the brain instead of being implanted in the grey matter. Their resolution signals are better than noninvasive BCIs. Their cranium brain tissue is responsible for deforming and deflecting signals. Also, they pose a lower risk of a scar tissue being formed in the brain as compared to a fully invasive BCIs.

They use Electrocorticography to measure the electrical activity of brain from beneath the skull. Daniel Moran and Eric Leuthardt of Washington University, St Louis tested this technology on humans in 2004. The researchers
They are implanted inside the skull but the rest of the

concluded that this technology allows rapid control and requires minimal training and is considered to provide perfect trade-off between level of invasiveness and signal fidelity. As compared to a scalp-recorded EEG, ECoG provides better signal- to noise ratio, higher spatial resolution, less training equipment and wider frequency range. Also, it poses low clinical risk and less technical difficulty. High control level with minimal training indicates its high potential to help people with motor disabilities.

Another technology involves light reactive imaging BCI, but still exist in theoretical world. A laser would be implanted in the skull. This laser is supposed to be trained on a single neuron. A sensor would measure the neuron's reflectance. The laser light pattern and wavelengths, the neuron reflects would change a little on its firing.

\section{Invasive}

These utilize recordings from activity of multiple neurons and ensembles of single brain cells. The origin of this technique are the experiments conducted by Fetz and colleagues in the 1960s and 1970s. The monkeys in these experiments achieved voluntary control of their cortical neurons, that were aided by biofeedback and thus could indicate firing rate of single neurons. A few years later, Edward Schmidt indicated that it is possible to extract voluntary motor commands from cortical neural activity and control prosthetic device that could help paralyzed patients in restoring their motor functions. Due to technical difficulties, it took almost two decades to test Schmidt's proposition. In 1995, there were experiments which led to evolution of BMI approaches which used long-term recordings from huge neuron population (100-400 units).

Invasive BCI aims to repair damaged sight and providing mobility to paralyzed patients. During neurosurgery, they are directly implanted into the brain's grey matter. Due to their implantation in the grey matter such devices create the BCI devices of highest quality signals. However, due to body's reaction to foreign object to an alien object in the brain, it can lead to scar-tissue build-up which can cause weak or even non-existent signals.

William Dobelle was a private researcher and one of the firsts to create a BCI to restore sight. He implanted his first prototype into a man called "Jerry". He was blinded in 1978 when he was an adult. It was a single-array BCI consisting of 68 electrodes. They were implanted onto his visual cortex and produced phosphenes which is responsible for the sensation of sight. The device would send signals with the help of cameras mounted on glass. In the beginning, Jerry could only see grey shades at a low frame-rate and had a restricted perception. He had to remain hooked to a mainframe computer to use the device but faster computers and evolution of computer into smaller devices provided him mobility. Dobelle introduced a second-generation implant for 16 paying patients. This device was a more advanced version. It enabled better mapping of phosphenes into coherent sight. Phosphenes were spread across the entire visual field. Researchers called this "the starry-night effect".
Published By:

Blue Eyes Intelligence Engineering and Sciences Publication

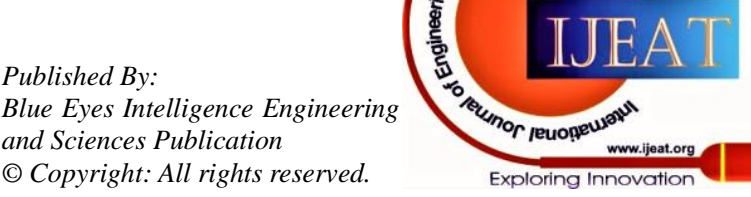


One of his patients, Jens, who was blinded as an adult, was successfully able to drive around the parking lot in research institute. Unfortunately, before his work could be documented, Dobelle died. Eventually, patients in the program had vision problems and lost their sight again.

Philip Kennedy and Roy Bakay led a team of researchers at Emory University, Atlanta and became the first to create a BCI and install into a human to produce high quality signals to simulate movement. Johnny Ray, their patient was suffering from 'locked-in syndrome'. Eventually, he learnt to control computer cursor. However, he died in 2002 of brain aneurysm. In 2005, tetraplegic Matt Nagle was able to control an artificial hand and became the first person to do so. They implanted 96-electrode BrainGate in his right precentral gyrus which is the region of motor cortex responsible for arm movement. He could also control lights, computer cursor and TV. Jonathan Wolpaw was awarded the Altran Foundation for innovation for development of a BCI that had electrodes on the skull surface instead of implanting them directly into human brain.

\section{PRINCIPLES OF BRAIN COMPUTER INTERFACE OPERATION}

Invasive BCIs depend on physiological properties subcortical and cortical neurons that are responsible for modulating their activity associated with their movements. Such modulations vary a lot with different trials and neurons. Therefore, even though, during the execution of a certain movement, neighbouring neurons might reveal a definite firing modulation pattern, a single neuron firing varies greatly with every trial.

The two essential, clinically viable operations expected from BCI are to extract the motor control signals from firing patterns of neuron population and the second is to use the control signals for reproducing motor behaviours in artificial actuators. BCI devices should feel the same way as the subject's own limbs. According to recent research, this might be achieved by constructing conditions where the brain experiences as experience-dependent plasticity and assimilate the prosthetic limb as a subject's body part. It was achieved through visual feedback. However, there is another methodology that is much more efficient involves using multiple artificial feedback signals that would be derived from position sensors and pressure that would be placed on the prosthetic limb. The brain would be trained by these feedback signals and to integrate properties of the prosthetic limb into tuning characteristics of cortical and sub-cortical neurons. To create a clinically viable BCI, the following bottlenecks should be passed:

1) Attain extensive recordings of huge number of neurons from various brain regions. This leads to development of a new variety of biocompatible 3D electrode matrices that generated thousands of channels of the recordings. There would be minimal inflammatory reaction and little tissue damage at implantation in the process.

2) Develop efficient algorithms to be assimilated in the software for BCI that could translate neuron activity into highly accurate command signals for controlling an artificial actuator containing multiple degrees of freedom.

3) Learn to utilize brain plasticity in order to include prosthetics in body representations.

Published By:

Blue Eyes Intelligence Engineering and Sciences Publication

(C) Copyright: All rights reserved.
4) Implement new generation prosthetics, that can effectively accept brain-derived control signals and perform movements with multiple degrees of freedom.

Some possible solutions to the above challenges are:

Modern technology offers implanted microwire arrays as the best compromise between recording longevity, safety and neuronal yield that is needed to run a BMI. Before it can be utilized for long-term applications, it needs to undergo some significant modifications such as biocompatibility, wireless head stages in order to amplify neuronal signals so that risk of infection can be reduced in the process of connecting external hardware to brain implants.

Recent microelectrode designs have made possible for good quality recordings to last several months and in some cases even years. However, the quality degrades due to electrode encapsulation by fibrous tissue and death of the cell near the electrode. Some approaches have been proposed by a few authors but their utility remains unclear. Some approaches would be electrodes coated with factors that would encourage neuronal growth or electrodes containing neurotrophic medium.

\section{INCORPORATING PROSTHETIC GADGETS INTO BODY REPRESENTATION}

Controlling an artificial actuator via BCI has been compared to the operation of operating tools. Head and Holmes have suggested that 'body schema' can widen itself to include a wielded tool.

Recent studies indicate that while manipulating a myoelectric prosthetic hand, the right ventral premotor cortex activated at specific points. The results suggested that long-term use of artificial actuator leads to sub-cortical and cortical mapping.

In another experiment, decoding algorithms were trained for prediction of limb movement of animals from their neural population. Their limbs stopped moving eventually as they were controlling actuators from their neuronal activity. Their neuronal tuning to their limb mobility decreased as they used their brain activity to control the artificial actuator.

\section{CREATING PROSTHETIC DEVICE TO FEEL LIKE PATIENT'S OWN LIMB}

Proprioceptive signals and peripheral tactile are responsible for our normal limb operation that gives us the sense that the limb is part of our body. We'll have to include sensors in the prosthetics that can send channels of sensory information back to the patient's brain, so that the prosthetic feels as a patient's body part. In recent BMIs animal subjects are sent sensory information via actuator by visual feedback. Motor parameters have predictions that are more stable in the presence of visual feedback than when it is absent.

Previous studies on monkeys have shown them sensing micro stimulation patterns and utilize these for guiding their behavioural responses. A recent study revealed that the owl monkeys could decode the vibratory stimuli that was applied on their arms and learn how to guide reaching movements.

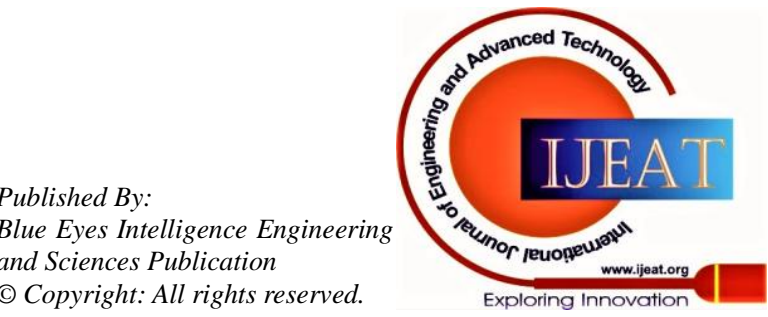




\section{Brain Computer Interface}

Later, matching patterns of micro stimulation through electrodes that were implanted in the primary somatosensory cortex were applied instead of the vibratory stimuli. Monkeys could interpret correctly all the instructions that cortical micro stimulation provided. Eventually, their behavioural performance surpassed their performance when they had vibratory stimulus applied to their skin.

Micro stimulation gives hope for development of a new generation of prosthetics restoring motor behaviours in patients.

\section{NON- ELECTROENCEPHALOGRAPHY BASED BRAIN COMPUTER INTERFACE}

The year 2016 saw the development of a new BCI that requires no visual fixation. It involves not fixing eyes on a particular letter on a virtual keyboard. The keyboard consists of letters having their own background circle that would in different time transitions micro-oscillate in brightness. Choice of letter selection is based on whatever fits best between background circle brightness oscillation pattern and oscillation pattern of unintentional pupil size. The user can improve his/ her accuracy by mental rehearsal of the words 'dark' and 'bright' in synchronization of transition of the circle or letter's brightness.

\section{ELECTROENCEPHALOGRAPHY BASED BRAIN COMPUTER INTERFACE}

EEG is considered to be the most studied of all non-invasive interfaces due to its portability, fine temporal resolution, low setup cost and ease of use. Bin $\mathrm{He}$ and his team at the University of Minnesota have recently suggested that EEG based BCIs could accomplish tasks nearly equivalent to invasive BCIs. Bin he and his team identified co-localization and co-variation of hemodynamic and electrophysiological signals that were induced by motor imagination. This was achieved by using functional neuroimaging including EEG source imaging and BOLD functional MRI. Bin He and his team showed the its potential to control flight of a virtual helicopter in 3-dimensional space based on motor imagination. In 2013, it was announced that he had developed the technology to guide a remote-control helicopter through an obstacle course.

\section{ELECTROCOTIGRAPHY}

ECoG or electrocorticography measures brain activity with the help of electrodes on the surface of the brain. ECoG has higher temporal and spatial resolution than EEG, but is an invasive technology that entails health hazard. Recent experiments on monkeys have revealed that ECoG performs with extreme accuracy for months. ECoG has been used on humans for analysing alpha, beta, gamma waves generated due to voluntary motor action.

\section{DRY ACTIVE ELECTRODE ARRAYS}

Babak Taheri at University of California, Davis in the early 1990s showed the first single, multichannel dry active electrode arrays by utilizing micromachining. In 1994, he published its construction and results. It was observed that this array electrode performed better than silver/ silver chloride electrodes. The device has four sensor sites with integrated electronics that reduces noise by utilizing the concept of impedance matching.

In 1999, Hunter Peckham and his team of researchers at Case Western Reserve University in Cleveland, ohio helped quadriplegic Jim Jatich by using 64-electrode EEG skullcap. Jim Jatich focused on simple, opposite concepts such as up and down. The researchers identified noise patterns by analysing his beta -rhythm EEG output using a software. They identified a pattern to control a switch. The pattern involved above average activity as on and below average activity as off. He was also enabled to control a computer cursor where they used signals to drive nerve controllers that were embedded in his hands.

\section{DIY BCI}

In 2001, a group of engineers and neuroscientists created a group called the OpenEEG Project. Their primary device was a ModularEEG. It consists of a 6-channel signal capture board. To build it at home, it would cost between $\$ 200$ and $\$ 400$. In 2010, a tutorial was published by the title of "How to Hack Toy EGGs" by the frontier Nerds of NYU's ITP program. It displayed how to create a single channel athome EEG with the help of Arduino and a mattel mindflex. In 2013, OpenBCI built a high quality and open source 8channel EEG acquisition board, sold under \$500. Two years later, they built Ultracortex, the first 3D printed headset and a Ganglion Board, a 4-channel EEG acquisition board which was sold for under $\$ 100$.

In 2015, NeuroTechX was introduced with the aim of creating an international network for nuerotechnology. It involves researchers, hackers and enthusiasts from different parts of the world.

Functional Magnetic resonance imaging (fMRI) and Magnetoencephalography and Magnetoencephalography (MEG) have been used in various non-invasive BCIs successfully. fMRI was used to achieve to scan two users to play real-time Pong by altering their blood flow or haemodynamic responses with the help of biofeedback methods.

In 2008, the Advanced Telecommunications Research (ATR) Computational Neuroscience Laboratories in Kyoto, Japan allowed scientists to directly reconstruct images from the brain and then display these images on a computer in black and white at a 10x10 pixel resolution.

In 2011, the researchers from UC Berkeley used fMRI data to publish a study of reconstruction of videos secondby-second that the subjects watched. The researchers built a statistical model that related the brain activity of subjects caused due to watching the videos to the visual pattern in the videos shown. This statistical model was then looked against 100 one-second video segments, in a million of seconds of random YouTube video database. These 100 video extracts which were all of one second were combined with a mashed-up image that looked similar to the video being viewed.

Published By:

Blue Eyes Intelligence Engineering and Sciences Publication

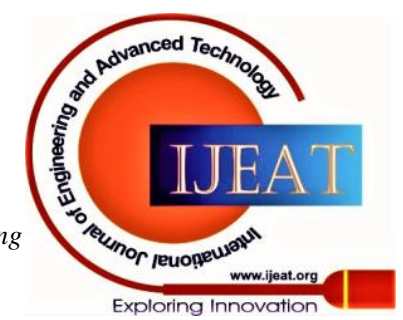




\section{BRAIN COMPUTER INTERFACE TECHNIQUES IN NEURO IMAGING}

Motor imagery comprises of imagining moving various body parts leading to sensorimotor cortex activation responsible for modulating sensorimotor oscillations in EEG. BCI would infer the user's intent. Motor imagery generally undergoes through a lot of training sessions before achieving the acceptable control of BCI. The users are unable to master control scheme, irrelevant of training duration. This leads to the pace of gameplay being very slow. Recently, advanced machine learning methods were developed for creating subject-specific model to detect performance of motor imagery. Mental relaxation of a subject is monitored by biofeedback. Various biofeedback systems have been used for treating disorders such as teeth grinding, attention deficit hyperactivity disorder (ADHD), sleep problems in children and chronic pain. Passive BCI enhance human computer interaction using BCI with the help of implicit knowledge on user's state. For example, simulations for detecting user's wish to push brakes while emergency car stopping process. Game developers that use passive BCIs have to recognize that as the user repeats game's levels, their cognitive state will adapt to it. The user will react in a different way to things from his first play to second play. For example, if the user is already expecting an event in the game, they'll be less surprised by it.

\section{WORKING}

BCI gives power to paralytic patients to control a wheelchair or write a book through thought alone. In June 2006, Peter Brunner, an American scientist demonstrated that he was able to compose a message by concentrating on a display. He wore a close-fitting cap that had electrodes embedded into it. The electrodes picked up EEG activity from his brain. This information was used to identify specific characters or letters for the message. His demonstration is based on the Wadsworth system. This system makes use of adaptive algorithms and pattern matching techniques to enhance communication.

\section{A. Background}

Brain cells use spikes or action potentials to communicate with each other. These are brief deviations of cell membrane potential from resting voltage. They reach other neurons while traveling along neuron's axons and long filaments.

\section{B. Electrode}

Usually electrodes of 40um to 200um diameters or more are implanted into the cortex. Electrodes used in BCIs are usually $1 \mathrm{~mm}$ in length and come in arrays. They pick up action potentials near the recording sites which usually occurs at <50um. Usually the uninsulated tip is the recording site.

\section{Brain signals}

BCIs record and decode brain signals. Neurons communicate by sending and receiving electrical signals. Advanced electrical sensors can listen to these signals. In bodies of healthy people, brain sends signals to muscles via the central nervous system. Medical conditions such as neuromuscular diseases can disrupt the communication between brain and muscles. This leads to paralysis. However, brain can generate activity for intended movements in many cases. BCI uses this brain activity to control assistive devices.

\section{Measuring brain signals}

There are many ways to measure brain signals. Electroencephalography (EEG) is one of the most commonly used techniques. This method requires electrodes to placed either directly under the scalp or in the brain tissue through a surgery. Signal quality from electrodes on surface of brain is better than those from electrodes from scalp. Electrodes on brain tissue do not cause any harm to the brain. Other methods include fMRI and MEG. fMRI uses MRI-scanner and MEG uses MEG-scanner for measuring brain activity. But they are not suitable for at-home use because they require huge expensive machines. Nearinfrared spectroscopy (NIRS) is another method for measuring brain activity. It does so by shining near-infrared light through skull. It does not require surgery and is portable. However, it's quality of measuring the brain activity is not enough.

\section{E. Brain function}

Brain has a 'control centre' for every body part, responsible for its movement. Different methods for measuring brain activity 'see' when these control centres are active. For example, waving your hand or wiggling toe are controlled by distinct brain regions. These control centres become active by simply thinking about moving a particular body part. Some area gets activated during numeric calculations, understanding language etc. The BCI can detect these areas. Thus, even a paralytic patient can perform mental tasks and therefore intentionally activate precise regions of brain, making BCIs a success.

\section{F. Brain signals}

Electrodes are placed on regions of brain that can be controlled. They receive signals that respond to control. The detected signals are converted to a command for operating a device. Thus, every time the patient counts backward in their head and select from a menu, for example an email program, they can click computer mouse.

\section{G. Signal production}

Subjects can produce brain signals in two ways:

1) Presenting stimuli to patient or having him/her imagine movements.

2) Reading brain activity of the subject

\section{H. Recording}

Signals are amplified about 10,000x in several stages. The head stage amplifier, located close to electrode array amplifies 1-10x along with doing high pass filtering. Second stage includes bandpass filtering and signal amplification is typically of $1000 x$ or more.

Published By:

Blue Eyes Intelligence Engineering and Sciences Publication

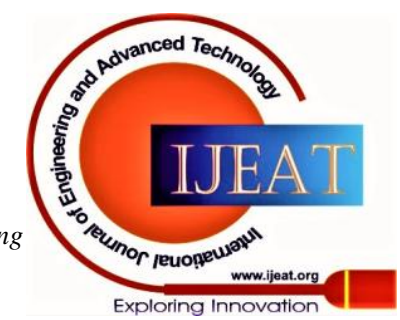




\section{Brain Computer Interface}

\section{Attention pattern detection}

EEG records brain signals on several frequencies. Different emotions correspond to different brainwave characteristics. For example, the brainwave of an alert person is different from a tired, slow person. Alpha, beta, gamma, delta, theta are different categories used to identify types of brainwaves. A person generates alpha waves while in meditation. They are more prominent in frequency range of 8 to $13 \mathrm{~Hz}$. Beta waves have frequency range of 13 to 22 $\mathrm{Hz}$ which changes when person is in a focused or excited state. Theta waves are generated when a person is in deep meditation and frequencies ranging from 4 to $8 \mathrm{~Hz}$. Delta waves have lowest frequency range of 1 to $4 \mathrm{~Hz}$. Gamma waves have frequency range above $32 \mathrm{~Hz}$.

\section{J. Signal processing}

After signal acquisition, the task of signal processing begins. It is processed by a single component consisting of 2 important steps: feature extraction and feature classification. Feature extraction involves evaluating signal and detecting signal features by using a signal extraction algorithm. The result is processed by a feature classification algorithm that relates the detected feature to the output device. This is a challenging process as the feature extraction algorithm has to deal with signal source which is noisy. To detect feature from such a source, researchers depend on temporal- spatial or time-frequency analysis.

\section{ETHICAL PERSPECTIVE}

Many ethical issues arise in the various applications of BCIs. Who would be held responsible if the brain-actuated mobile robot causes damage? Is it degrading our human dignity by using pre-conscious brain information processed by a BCI? Should young people be allowed to interact BCI based games with their plastic brain? Is user personality affected by use of BCIs?

BCIs face a wide array of issues such as privacy, safety, oral responsibility. Before participating in a study, the subject should be informed of all relevant risks, benefits, purpose of research and procedure. All misunderstandings should be clarified.

\section{NEUROSURGICAL ISSUES}

The most important question is the safety of BCI. It should be reliable and durable. Patients response is also important, for example after a period of time, will signal acquisition face obstruction from scar formation. If the BCI has a short half-life, it will require reimplantation after short-time frames that risks injury to patient in that area.

For a BCI to enable a motor impaired patient, it requires a minimum of three- dimensional control. One dimensional control allows only binary interaction. Two-dimensional control allows cursor to move along $\mathrm{x}$ and $\mathrm{y}$ axis. However, to be able to control a robotic arm, many more DOF are required. Speed and accuracy of the device are two major factors to be considered so that the patient can function with minimum errors in real-time.

\section{MENS REA}

In Mens Rea, user commits the crime with a certain mental state. Active BCIs do not affect the user's intentions. According to the American Model Penal code, for some crime the agent must act purposely while for others recklessly to be found guilty.

\section{ACTUS REUS}

Agent is found guilty only if he/she satisfies a specific conduct, status or omission.

Actus Reus is satisfied if the agent does not perform some obligated task or performs a task, he/she ought not to or becomes member of a prohibited group such as a terrorist group.

\section{TYPOLOGY}

\section{A. Active}

User performs a mental task deliberately to generate a particular pattern of brain activity. Imagining movement of different parts of the body activates different parts of motor cortical areas. However, it is prone to errors and requires a lot of effort.

\section{B. Reactive}

It involves modulating brain activity in response to an external stimulus. For example, in P300-based selection, stimuli like letters or symbols or other characters are displayed on a screen. User directs his/ her attention to the intended symbol. The BCI device identifies the signal generated about $300 \mathrm{~ms}$ after the stimulus is flashed on the screen. Locked-in patients can create art with the help of Brain Painting application.

\section{Passive}

It monitors the brain activity of patient. The patient does not need to perform a mental task. They monitor workload and arousal, thus preventing risky situations by identifying user's lapse in attention. Affective BCIs might detect that the user is frustrated or bored and adapt a suitable task or introduce engaging elements

\section{BRAIN STIMULATION IN NEUROREHABILITATION OF STROKE}

One of the major causes of chronic disability is stoke and neurotrauma. Electric currents have been shown to modulate cognition and behaviour. It was demonstrated that tDCS or transcranial direct current stimulation can help in improving learning ability. When experimented on chronic stroke patients, their reaction time and pinch force of affected hand improved. Many studies revealed that tDCS timing relative to training affects stimulation effects. Recently, tDCS and EEG-based BMI were combined. It involved placing stimulation electrode to only $1 \mathrm{~cm}$ near EEG electrode, being used for BMI control. This was done to avoid direct contact as it could lead to amplifier saturation.

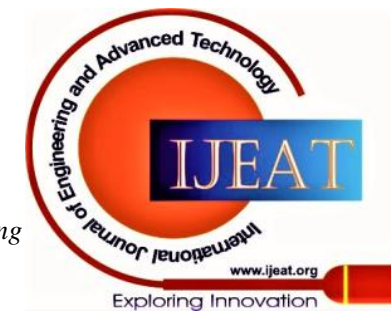


Another strategy was used that involves use of neuromagnetic brain signals (MEG). It could pass through stimulation electrode. This revealed that BMI utilizing SMR or primary motor cortex could control orthotic device of this region.

\section{APPLICATIONS}

\section{A. Medical application:}

Studies on consciousness level determination systems with their brain-related studies have been developed. The significance of these studies is the decrease in alertness level that results from alcohol drinking and smoking.

Traffic accidents are one of the main causes for death and serious injuries. Researchers have studied motion sickness in drivers. Motion sickness leads to loss of driver's ability to maintain self-control which leads to accident. In a study, a virtual reality-based motion sickness platform with a 32channel EEG system and joystick was designed for reporting motion sickness level in experiments.

BCIs have been used to detect and diagnose disorders such as brain tumour, dyslexia, narcolepsy, epilepsy and encephalitic.

\section{B. Smart environment}

Combination of Internet of things and BCIs offer luxury and safety. Brain computer interface-based smart living environmental auto-adjustment control system (BSLEACS) has been proposed to monitor user's mental state and adapt to surrounding appliances. Brain signals improve work conditions by analysing mental fatigue and task time in EEG features.

\section{Games and entertainment}

Combination of BCIs with games provides a multi-brain entertainment experience. In a video game called BrainArena, users can join a competitive football game with the help of BCIs. They can score goals by hand movements. An EEG game for emotional control has been developed in which the player can move the ball only if he/she is relaxed. Therefore, the most relaxed player wins and thus they need to control their stress in order to win at the game.

\section{Security and authentication}

Security systems that are object or biometric based are prone to vulnerable to several attacks such as shoulder surfing and theft crime. Cognitive biometrics use brain signals for identifying information. An external observer cannot attain brain signal. They are of importance to patients who are disabled and do not have the required physical trait.

\section{E. Opportunistic state-based detection}

Neural state monitoring in combination with pervasive intelligence has the potential to change our surrounding environment. Early symptoms of ailments like migraines could help the patient in getting recommendations from a physician to stop a particular activity or other factors to prevent those symptoms.

Most alarm clocks go off without any regard of the sleep stage, the user is in. A person's energy level during the day depends on the sleep stage they were in before waking up. By involving a device that incorporates an interactive system with the brain activity such that the device would wake the user when they are in the optimal sleep cycle. Thus, the user would wake up refreshed and alert.

\section{F. Use of speech for brain computer interface control}

Speech processing has been studied using various neuroimaging, lesion models, neurophysiological studies. These studies have revealed the that speech processing has a distributed network of cortical areas including the Wernicke and Broca centre. Wernicke centre is responsible for receptive language whereas the Broca centre is associated with expressive language.

Since there are many cortical areas related to speech, separable physiology and cognitive task of using speech may provide features to motor derived control.

Wilson showed that auditory cortex can be utilized for controlling computer cursor. A recent study consisting of 9 participants, showed that ECoG signals differentiate between speech or imagined speech and rest.

\section{CHALLENGES}

Current BCIs have the potential to detect only 2-3 mental states. One of the main challenges is that signals acquired by the same person during different sessions vary. Also, different users executing same tasks generate different EEG signals. The signals acquired contains noise such as eye and muscle movements. These are called artifacts and are removed manually by clinicians.

\section{FUTURE}

Many studies have been done in this field which continues to develop and promises a great future. Eventually, they may be used in severely disabled people to restore their motor functions. BCIs depend on sensors and other hardware for signal acquisition. They are expected to be small and portable and not require skin abrasion. They must be easy to set-up and function for many hours without maintenance requirement. Most of these needs are met by current technology. Dry electrode options are also available nowadays. The components need to be able to record signals for years, remain reliable and functional and needs to be safe. Although, many advances are being made in this filed. It is unclear which technology would be more successful. ECoG might prove to be more stable than BCIs that are based on neuronal action potentials. Important researches continue to be experimented on animals before human trials begin. Even after many years of research and development, BCIs have not achieved great success. Expectations regarding its applications are high. Clear distinction should be made between currently viable and potential applications to avoid unrealistic expectations During experiments, applying stimulus to subjects that rely on presence or absence of a certain brain activity can allow for development of complex designs. Recent studies in singletrial analysis have seen an increased interest in brain reading, where contents of subjective perception are inferred.

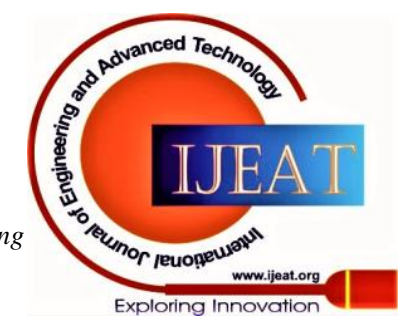




\section{Brain Computer Interface}

Focus on single- trial analysis will eventually increase our knowledge of human cognition

\section{RESULT AND DISCUSSION}

In this paper, we have presented studies and demonstrations of various types of Brain Computer Interface and its results done over the past few decades. These studies and experiments have helped people with disabilities such as paralysis, stroke etc. with an opportunity to communicate to the outer world. The studies involve working of the brain computer interface and its applications in the life of both disabled and healthy people. We discussed its successful collaboration with Internet of things (IOT) and its impact on security and authentication procedures, games and entertainment field and in the medical field. Early experiments were mostly done on animals. Later they were done on tetraplegic patients.

Some researches have considered the problem of long training durations and successfully decreased it to some extent. This has helped motor- impaired patients to carry out daily activities such as sending and reading emails and controlling a wheel chair.

\section{CONCLUSION}

Our vision of neuro prosthetics that might become a reality in the next 10-20 years has a fully- implantable recording system that can transmit multiple streams of electric signals wirelessly. These signals are derived from thousands of neurons and sent to BMI that can decode temporal and spatial characteristics of mobility, also including cognitive characteristics of intended actions. The device would merge peripheral low-level control signals that would be derived from an artificial 'reflex-like' control loop and high-order motor commands that would be derived from subcortical and cortical neural activity. Artificial actuators with many arrays of position and touch sensors would be able to generate streams of sensory feedback signals directly delivered through spatiotemporal patterns of multi-channel micro stimulation, to subcortical and cortical somatosensory region of subject's brain. It's been suggested that by 2050 , BCI would be helping humans control object by using their mind. BCIs are involved in various research fields such as neuromarketing and advertising, games and entertainment, medical and security and authentication. It can have a great impact in the field of medicine and rehabilitation. However, it faces challenges such as minimizing cost, identify suitable patient selection and more clinical trials to test its efficacy. Patient's needs and priorities are critical so that the prosthetic device feel like a part of their own body.

\section{ACKNOWLEDGMENT}

This paper would not have been possible without the support of my Mentor, Prof. Shalini L from School of Computer Science of Vellore institute of technology, Vellore, India. Her knowledge and constant guidance have assisted me in being able to research on one of the most concerned problems.

\section{REFERENCES}

1. Akhilesh Bhagwani, Chitransh Sengar, Jyotsna Talwaniper, Shaan Shama, "Human Computer Interaction", Intemational Joumal of Advancements in Research and Technology, Volume 1, Issue 3, August-2012. Available: academia.edu/2236651/Human_Computer_Interaction

2. Lebedev MA,Nicolelis MA,'Brain-machine interfaces: Past, present and future", Trends Neurosci,2006;29(9):535-546 doi:10.1016/j.tins.2006.07.004, Available: pubmed. ncbi. nlm. nih. gov/16859758

3. Omkar Sahasrabudhe," Brain Computer Interface", International Journal of Engineering Technology Research and Management, Vol(03), Issue(03), Available: https://www. academia. edu/38776926/BRAIN_COMPUTER_INTERFACE

4. Sarah N. Abdulkar, Ayman Atia, Mostafa-Sami M. Mostafa, Brain computer interfacing: applications and challenges,Egpt. Informat. J. 16(2) (2015) 213-230, Available:

https://www.sciencedirect.com/science/article/pii/S11108 66515000237

5. Felix G. Hamza-Lup, Aditya Suri, Ionut E. Iacob, Iona R. Goldbach, Lateef Rasheed, Paul N. Borza, Attention Patterns Detection using Brain Computer Interfaces, arXiv:2005.11151, Available: arxiv.org/abs/2005.11151

6. Oliver Tonet, Martina Marinelli, Luca Citi, Paolo Maria Rossini,Luca Rossini,Giuseppe Megali, Paolo Dario, Defining brain-machine interface applications by matching interface performance with device requirements, Journal of Neuroscience Methods,2008 Jan;167(1):91104. DOI: 10.1016/j.jneumeth.2007.03.015, Available: repository.essex.ac.uk/8793/1/Tonet2008DefBMIApplicMergingIntPe rfDevicRequir.pdf

7. Sujo R. Soekadar, Niels Birbaumer, Marc W. Slutzky, Leonardo G. Cohen, Brain machine interfaces in neurorehabilitation of stroke, Soekadar, S.R,et al,Brain-machine interfaces in neurohabilitation of stroke,Neurobiol.Dis.(2014),Available:http://dx.doi.org/10.1016/j.nbd .2014.11.025

8. Ulrich Hoffmann, Jean-Marc Vesin, Touradj Ebrahimi, Recent Advances in Brain-Computer Interfaces, (2007) ,10.1109/MMSP.2007. 4412807, Available:researchgate.net/publication/37452383_Recent_Advances_i n_Brain-Computer_Interfaces

9. Rak, Remigiusz \& Kołodziej, Marcin \& Majkowski, Andrzej. (2012). Brain-Computer Interface as measurement and control system the review paper. Metrology and Measurement Systems. 19. 427-444. Available:

https://www.researchgate.net/publication/258509662 BrainComputer Interface as measurement and control system the revie W_paper

10. Shih, J.J., Krusienski, D.J., \& Wolpaw,J.R. (2012). Brain-computer interfaces in medicine. Mayo Clinic proceedings, 87(3), 268 279.Available: https://doi.org/10.1016/j.mayocp.2011.12.008

11. Leuthardt, E. C., Schalk, G., Moran, D., \& Ojemann, J. G. (2006). The emerging world of motor neuroprosthetics: a neurosurgical perspective. Neurosurgery, 59(1), 1-14 Available: https://doi.org/10.1227/01.NEU.0000221506.06947.AC

12. A. Nijholt et al., "Brain-Computer Interfacing for Intelligent Systems," in IEEE Intelligent Systems, vol. 23, no. 3, pp. 72-79, MayJune 2008, doi: 10.1109/MIS.2008.41.Available: https://ieeexplore.ieee.org/document/4525145/authors\#authors

13. Ojemann, Jeffrey \& Leuthardt, Eric \& Miller, Kai. (2007). Brainmachine interface: restoring neurological function through bioengineering. Clinical neurosurgery. 54. 134-6.Available: https://www.researchgate.net/publication/51393494_Brainmachine_interface_restoring_neurological_function_through_bioengi neering

14. Leuthardt EC, Schalk G, Roland J, Rouse A, Moran DW. Evolution of brain-computer interfaces: going beyond classic motor physiology. Neurosurg Focus. 2009;27(1):E4 doi:10.3171/2009.4.FOCUS0979.Available: https://www.academia.edu/27390219/Evolution of brain computer _i nterfaces going beyond classic motor physiology

15. Ortiz-Rosario A, Adeli H. Brain-computer interface technologies: from signal to action. Reviews in the Neurosciences. 2013 ;24(5):537552. DOI: 10.1515/revneuro-2013-0032. Available:

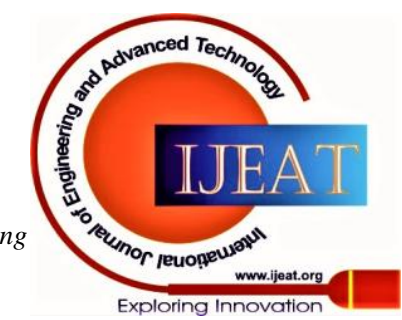


https://www.academia.edu/7248773/Brain_computer_interface_techn ologies_from_signal to action

16. van Gerven, M., Farquhar, J., Schaefer, R., Vlek, R., Geuze, J., Nijholt, A., Ramsey, N., Haselager, P., Vuurpijl, L., Gielen, S., \& Desain, P. (2009). The brain-computer interface cycle. Journal of neural engineering, 6(4), $041001 . \quad$ Available: https://www.researchgate.net/publication/26686434_The_braincomputer_interface_cycle

17. Lance, Brent \& Kerick, Scott \& Ries, Anthony \& Oie, Kelvin \& McDowell, Kaleb. (2012). Brain Computer Interface Technologies in the Coming Decades. Proceedings of the IEEE. 100. 10.1109/JPROC.2012.2184830.

Available:https://ieeexplore.ieee.org/stamp/stamp.jsp?arnumber=6162 $\underline{941}$

18. Vuckovic A. (2009). Non-invasive BCI: how far can we get with motor imagination? Clinical neurophysiology: official journal of the International Federation of Clinical Neurophysiology, 120(8), 1422 1423.

Available: https://www.academia.edu/21173386/Non_invasive_BCI_How_far_c an_we get_with_motor_imagination

19. Graimann B., Allison B., Pfurtscheller G. (2009) Brain-Compute Interfaces: A Gentle Introduction. In: Graimann B., Pfurtscheller G., Allison B. (eds) Brain-Computer Interfaces. The Frontiers Collection. Springer, Berlin, Heidelberg. Available: https://doi.org/10.1007/978-3-642-02091-9_1

20. Soman, Sumit \& Sen Gupta, Soumya \& Raj, P Govind. (2012). Non Invasive Brain Computer Interface for Controlling User Desktop.Available:

https://www.researchgate.net/publication/260350225_Non_Invasive Brain_Computer_Interface_for_Controlling_User_Desktop

21. Tamburrini, Guglielmo. (2009). Brain to Computer Communication: Ethical Perspectives on Interaction Models. Neuroethics. 2. 137-149. 10.1007/s12152-009-9040-1. Available: https://www.researchgate.net/publication/225735446 Brain to Comp uter Communication Ethical Perspectives on Interaction Models

22. Schalk G. (2008). Brain-computer symbiosis. J. Neural Eng. 5 1-15. 10.1088/1741-2560/5/1/P01

Available: https://www.ncbi.nlm.nih.gov/pmc/articles/PMC2722922/

23. Otal, Begonya \& Vargiu, Eloisa \& Miralles, Felip. (2014). Towards BCI Cognitive Stimulation: From Bottlenecks to Opportunities. 10.3217/978-3-85125-378-8-53.

Available: https://www.researchgate.net/publication/274252649_Towards_BCI_ Cognitive_Stimulation_From_Bottlenecks_to_Opportunities

24. Nicolas-Alonso, L. F., \& Gomez-Gil, J. (2012). Brain compute interfaces, a review. Sensors (Basel, Switzerland), 12(2), 1211-1279. Available: https://www.ncbi.nlm.nih.gov/pmc/articles/PMC3304110/

25. Tyagi, A. and Nehra, V.(2013) 'Brain-computer interface: a thought translation device tuming fantasy

into

reality', Int. J. Biomedical Engineering and Technology,Vol. 11, No. 2, pp.197-211.

26. Ahn, M., Lee, M., Choi, J., \& Jun, S. C. (2014). A review of braincomputer interface games and an opinion survey from researchers, developers and users. Sensors (Basel, Switzerland), 14(8), 1460114633.

https://www.ncbi.nlm.nih.gov/pmc/articles/PMC4178978/

Available:

27. Fabien Lotte, Laurent Bougrain, Maureen Clerc. Electroencephalography (EEG)-based BrainComputer Interfaces. Wiley Encyclopedia of Electrical and Electronics Engineering, Wiley, pp.44, 2015, ff10.1002/047134608X.W8278ff. ffhal-01167515. Available: https://hal.inria.fr/hal-01167515/document

28. Fabien Lotte, Chang S. Nam, Anton Nijholt. Introduction: Evolution of Brain-Computer Interfaces. Chang S. Nam; Anton Nijholt; Fabien Lotte. Brain-Computer Interfaces Handbook: Technological and Theoretical Advance, Taylor \& Francis (CRC Press), pp.1-11, 2018 9781498773430. ffhal-01656743. Available: https://hal.inria.fr/hal01656743/document

29. Clemens Brunner, Niels Birbaumer, Benjamin Blankertz, Christoph Guger, Andrea Kübler, Donatella Mattia, José del R. Millán, Felip Miralles, Anton Nijholt, Eloy Opisso, Nick Ramsey, Patric Salomon \& Gernot R. Müller-Putz (2015) BNCI Horizon 2020: towards a roadmap for the BCI community, Brain-Computer Interfaces, 2:1, 110, DOI: 10.1080/2326263X.2015.1008956

Available: https://www.tandfonline.com/doi/full/10.1080/2326263X.2015.10089 $\underline{56}$

30. Schwartz, Andrew \& Cui, Xinyan \& Weber, Douglas \& Moran, Daniel. (2006). Brain-Controlled Interfaces: Movement Restoration with Neural Prosthetics. Neuron. $52.205-20$. 10.1016/j.neuron.2006.09.019. Available: https://www.sciencedirect.com/science/article/pii/S089662730600726 4
Published By:

Blue Eyes Intelligence Engineering and Sciences Publication (C) Copyright: All rights reserved.
31. Suleiman, Abdul-bary \& Suleiman, Abdul-Bary \& Fatehi, AbdulHameed \& A. Fathi, Toka. (2013). FEATURES EXTRACTION TECHNIQES OF EEG SIGNAL FOR BCI APPLICATIONS FEATURES EXTRACTION TECHNIQES OF EEG SIGNAL FOR BCI APPLICATIONS. Available: https://www.researchgate.net/publication/332606126 FEATURES E XTRACTION_TECHNIQES_OF_EEG_SIGNAL_FOR_BCI_APPLI CATIONS_FEATURES_EXTRACTION_TECHNIQES_OF_EEG_S IGNAL FOR BCI_APPLICATIONS

32. Ramadan, Rabie \& Refat, Samah \& Elshahed, Marwa \& Ali, Rasha (2015). Basics of Brain Computer Interface. Intelligent Systems Reference Library. 74. 31-50. 10.1007/978-3-319-10978-7_2. Available:

https://www.academia.edu/32721541/Basics of Brain Computer Int erface \%C3\%81 Systems of BCI \%C3\%81 BCI monitoring hard ware and software \%C3\%81_BCI trends

33. Haselager, P., et al. A note on ethical aspects of BCI. Neural Networks (2009), doi:10.1016/j.neunet.2009.06.046. Available: https://bmcmedethics.biomedcentral.com/articles/10.1186/s12910017-0220-y

34. Siddiqui, Mariam \& Khalidi, Ayman \& Farooq, Omar \& Khan, Yusuf. (2011). EXECUTED WRIST MOVEMENT CLASSIFICATION FOR BRAIN COMPUTER INTERFACE. Available: https://www.academia.edu/21778247/feature extraction_of executed _wrist_movement_using_BCI

35. Vlek, R. J., Steines, D., Szibbo, D., Kübler, A., Schneider, M. J., Haselager, P., \& Nijboer, F. (2012). Ethical issues in brain-compute interface research, development, and dissemination. Journal of neurologic physical therapy : JNPT, 36(2), 94-99. https://doi.org/10.1097/NPT.0b013e31825064cc. Available: https://www.researchgate.net/publication/224971384_Ethical_Issues in_Brain-

Computer_Interface_Research_Development_and_Dissemination

36. McCullagh, P., Lightbody, G., Zygierewicz, J. et al. Ethica Challenges Associated with the Development and Deployment of Brain Computer Interface Technology. Neuroethics 7, 109-122 (2014). Available: https://doi.org/10.1007/s12152-013-9188-6

37. Bockbrader, Marcie \& Francisco, Gerard \& Lee, Ray \& Olson, Jared \& Solinsky, Ryan \& Boninger, Michael. (2018). Brain Computer Interfaces In Rehabilitation Medicine. PM\&R. 10 10.1016/j.pmrj.2018.05.028. https://onlinelibrary.wiley.com/doi/10.1016/j.pmrj.2018.05.028

38. AlZoubi, Omar \& Koprinska, Irena \& Calvo, Rafael. (2008) Classification of Brain-Computer Interface Data. 7th Australasian Data Mining Conference. 87. 123-131. Available: https://crpit.scem.westernsydney.edu.au/confpapers/CRPITV87AlZou bi.pdf

39. Steinert, S., Friedrich, O. Wired Emotions: Ethical Issues of Affective Brain-Computer Interfaces. Sci Eng Ethics 26, 351-367 (2020). Available:https://doi.org/10.1007/s11948-019-00087-2

40. Soekadar, S.R., Birbaumer, N., \& Cohen, L.G. (2011). Braincomputer-interfaces in the rehabilitation of stroke and neurotrauma. Available:

https://www.academia.edu/20495440/Brain Computer Interfaces in the Rehabilitation_of_Stroke_and_Neurotrauma

41. Birbaumer, Niels \& Gallegos, Guillermo \& Wildgruber, Moritz \& Silvoni, Stefano \& Soekadar, Surjo. (2013). Direct Brain Control and Communication in Paralysis. Brain topography. 27. 10.1007/s10548013-0282-1.

Available: https://www.academia.edu/20495401/Direct_Brain_Control_and_Co mmunication in Paralysis

42. Mikołajewska, E., Mikołajewski, D. Ethical considerations in the use of brain-computer interfaces. cent.eur.j.med 8, 720-724 (2013) Available: https://doi.org/10.2478/s11536-013-0210-5

43. Klein, Courtney. (2012). Guilty Act, Guilty Mind: establishing Actus Reus and Mens Rea in situations of conspiracy and accessory.Available:

https://www.researchgate.net/publication/261026128_Guilty_Act_Gui lty Mind establishing Actus Reus and Mens Rea in situations of conspiracy and accessory

44. Donoghue, John. (2002). Donoghue, J.P. Connecting cortex to machines: recent advances in brain interfaces. Nat. Neurosci. 5, 10851088. Nature neuroscience. 5 Suppl. 1085-8. 10.1038/nn947. Avaialble:

https://www.researchgate.net/publication/11060047_Donoghue_JP_C

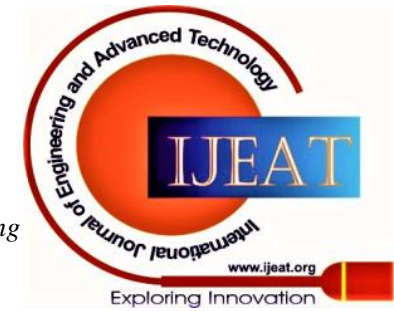




\section{Brain Computer Interface}

onnecting_cortex to_machines_recent_advances_in_brain_interfaces

Nat_Neurosci_5_1085-1088

45. Steinert, S., Bublitz, C., Jox, R. et al. Doing Things with Thoughts: Brain-Computer Interfaces and Disembodied Agency. Philos. Technol. 32, 457-482 (2019). Available: https://doi.org/10.1007/s13347-018-0308-4

46. Thompson, K. Committing Crimes with BCIs: How Brain-Computer Interface Users can Satisfy Actus Reus and be Criminally Responsible. Neuroethics (2019). https://doi.org/10.1007/s12152-019-09416-5

47. Nijholt, Anton \& Erp, Jan \& Heylen, Dirk. (2008). BrainGain: BCI for HCI and Games. International Journal of Autonomous and Adaptive Communications Systems. Available: https://www.researchgate.net/publication/228607607 BrainGain BCI for HCI and Games

48. Kübler, A., Kotchoubey, B., Kaiser, J., Wolpaw, J. R., \& Birbaumer, N. (2001). Brain-computer communication: unlocking the locked in. Psychological bulletin, 127(3), 358-375. Available: https://doi.org/10.1037/0033-2909.127.3.358

49. Padfield, N., Zabalza, J., Zhao, H., Masero, V., \& Ren, J. (2019). EEG-Based Brain-Computer Interfaces Using Motor-Imagery: Techniques and Challenges. Sensors (Basel, Switzerland), 19(6), 1423. Available: https://doi.org/10.3390/s19061423

50. Allison BZ, Wolpaw EW, Wolpaw JR. Brain-computer interface systems: progress and prospects. Expert Rev Med Devices. 2007;4(4):463-474. doi:10.1586/17434440.4.4.463Available: https://www.neurotechcenter.org/sites/default/files/misc/allison2007.p df

\section{AUTHOR'S PROFILE}

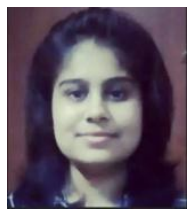

Vanshi Sharma was born in Bharatpur, Rajasthan India on $7^{\text {th }}$ August, 1999. She completed her $10^{\text {th }}$ and $12^{\text {th }}$ grade from Maharaja Sawai Man Singh Vidyalaya, Jaipur, India. She is currently pursuing her Bachelor's degree in Computer Science Engineering at Vellore institute of technology, Vellore, Tamil Nadu, India. She is a member of ' Students for Exploration and Development of Space (SEDS)' and ' Institute of Electrical and Electronics Engineers(IEEE-CS)' She has previously worked on projects involving cyber security, image processing and blockchain. She is currently working on projects which involve concepts of Natural language processing, Artificial Intelligence, and Machine learning. 